\title{
Bone metastasis from cholangiocarcinoma mimicking osteosarcoma: A case report and review literature
}

\author{
PRIN CHINDAPRASIRT ${ }^{1}$, JULALUCK PROMSORN ${ }^{1}$, PITI UNGAREEWITTAYA ${ }^{2}$, \\ NATTAPHON TWINPRAI ${ }^{3}$ and JARIN CHINDAPRASIRT ${ }^{4}$ \\ Departments of ${ }^{1}$ Radiology, ${ }^{2}$ Pathology and ${ }^{3}$ Orthopedic Surgery; ${ }^{4}$ Division of Oncology, \\ Department of Internal Medicine, Faculty of Medicine, Khon Kaen University, Khon Kaen 40002, Thailand
}

Received August 1, 2018; Accepted September 17, 2018

DOI: $10.3892 / \mathrm{mco} .2018 .1720$

\begin{abstract}
Cholangiocarcinoma is an aggressive tumor of the hepatic biliary system and it commonly spreads to the regional lymph nodes, liver and lungs. However, bone metastasis from cholangiocarcinoma is rare compared with other tumors. We herein present the case of a 61-year-old Asian woman who presented with pain in the right scapular area. Magnetic resonance imaging revealed bone destruction and an adjacent soft tissue mass at the right scapula. The findings on computed tomography imaging were compatible with cholangiocarcinoma. Bone biopsy was performed and the diagnosis of cholangiocarcinoma with bone metastasis was confirmed. The survival time was 10 months, despite administration of palliative radiotherapy and chemotherapy. Therefore, bone metastasis from cholangiocarcinoma should be considered as a differential diagnosis in patients who present with an osteolytic bone lesion and a liver mass.
\end{abstract}

\section{Introduction}

Cholangiocarcinoma (CCA) is a malignant tumor that originates from the epithelium of the bile ducts. Jaundice and abdominal pain are the most common presentations. The tumor commonly metastasizes via lymphatic spread to the regional lymph nodes, followed by hematogenous metastasis to the liver, lungs and peritoneum (1). Distant metastasis is uncommon, although bone, muscle and brain metastasis have also been reported (2-5). Patients usually develop distant metastasis in the late stages of the disease.

Appendicular bone metastasis from CCA as a first presentation is an extremely rare event, considering the very small number of published case reports. Bone scan is not usually routinely performed in all patients first diagnosed with

Correspondence to: Dr Jarin Chindaprasirt, Division of Oncology, Department of Internal Medicine, Faculty of Medicine, Khon Kaen University, Khon Kaen 40002, Thailand

E-mail: jarich@kku.ac.th

Key words: biliary tract neoplasm, cholangiocarcinoma, bone metastasis
CCA (6), but only in those presenting with bone pain and pathological fractures.

The aim of the present study was to report a case of CCA with multiple osseous metastases, with the imaging findings (severe cortical bone destruction and a large soft tissue mass) mimicking osteosarcoma.

\section{Case report}

A 61-year-old female with a history of well-controlled hypertension presented on September 2016 to the Khon Kaen University Hospital (Khon Kaen, Thailand) with a history of pain in the right scapular area for 5 months. The pain was described as dull aching, and was more severe at night. Two months prior to visiting our hospital, the patient noticed a lump in the right side of her back, gradually increasing in size. She started to feel numbness in her right arm and forearm, and reported a history of asthenia for $\sim 1$ month. On physical examination, the patient was not icteric, with impalpable cervical and supraclavicular lymph nodes. The liver was found to be mildly enlarged, with no chronic liver stigmata. A $12-\mathrm{cm}$ mass was identified in the right shoulder area.

A magnetic resonance imaging examination of the right shoulder revealed aggressive osseous destruction of nearly the entire right scapula, with an adjacent extraosseous soft tissue mass encasing the neurovascular bundle (Fig. 1A). Bone scintigraphy also demonstrated multiple bone metastases to the skull, mid-cervical vertebrae, T4, L1-2 and L4-5 vertebrae, pelvis, left proximal femur, right scapula, right humerus, proximal right forearm, lateral aspect of the right 8th rib and posterior aspect of the right 9 th rib (Fig. 1B). A computed tomography scan of the whole abdomen revealed an ill-defined hypodense lesion at hepatic segment 7/6 with subcapsular retraction, compatible with CCA (Fig. 1C).

A biopsy of the scapular mass was performed, and the histological examination revealed bone invasion by neoplastic cells arranged in cords and glandular formations, with a desmoplastic stromal reaction, resembling metastatic adenocarcinoma (Fig. 2). Immunohistochemical examination revealed that the tumor cells were positive for cytokeratin (CK)7, CK20 and carbohydrate antigen 19-9, and negative for thyroid transcription factor-1, confirming the diagnosis of CCA. 

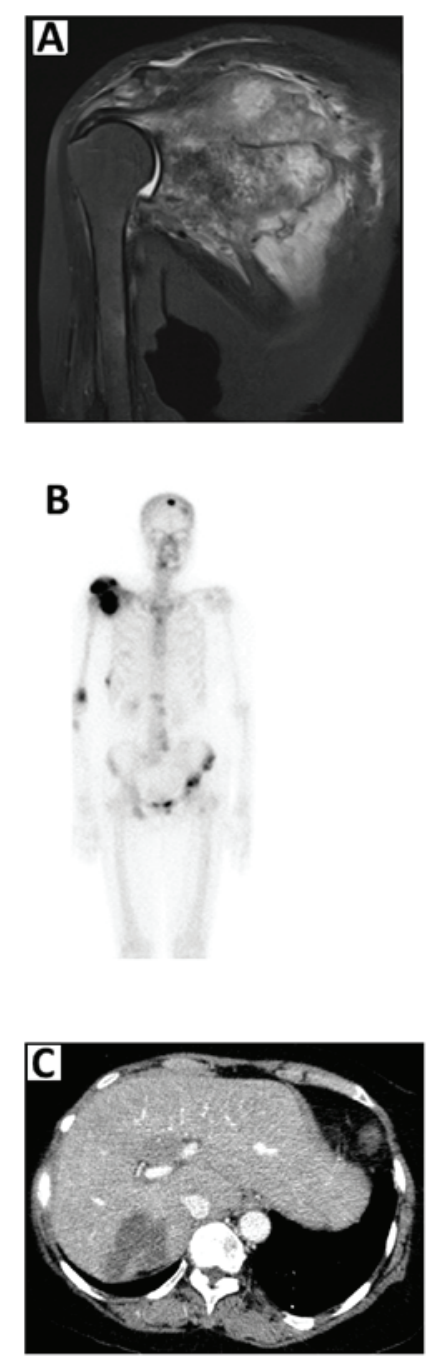

Figure 1. (A) Magnetic resonance imaging examination of the right shoulder showing destruction of nearly the entire right scapula, with an adjacent extraosseous soft tissue mass encasing the neurovascular bundle. (B) Bone scintigraphy revealed multiple bone metastases to the skull, mid-cervical vertebrae, T4, L1-2 and L4-5 vertebrae, pelvis, left proximal femur, right scapula, right humerus, proximal right forearm, lateral aspect of the right 8th rib and posterior aspect of the right 9 th rib. (C) A computed tomography scan of the whole abdomen revealed an ill-defined hypodense lesion at hepatic segment $7 / 6$ with subcapsular retraction, compatible with cholangiocarcinoma.

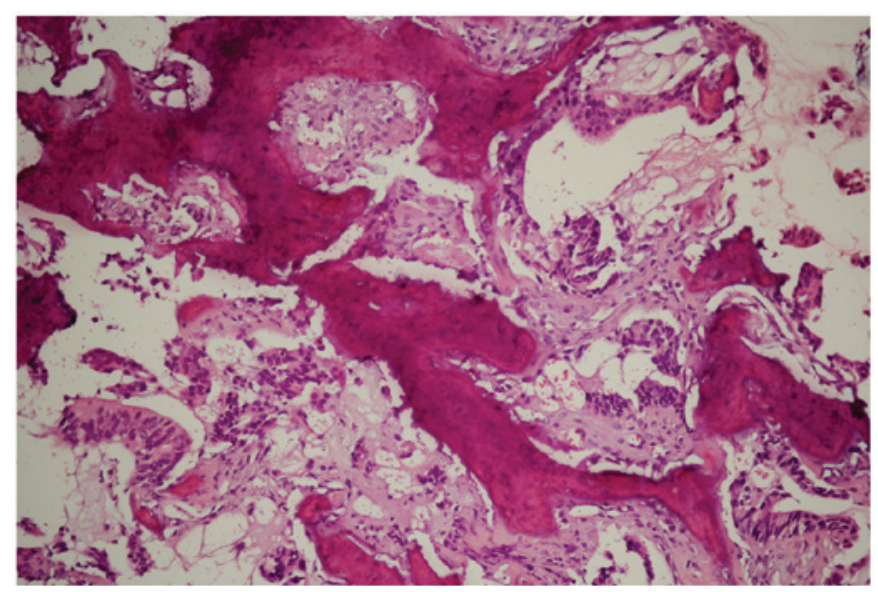

Figure 2. A histological examination of the scapular mass revealed bone invasion by neoplastic cells arranged in cords and glandular formations, with a desmoplastic stromal reaction, resembling metastatic adenocarcinoma. Hematoxylin and eosin staining; magnification, x200.

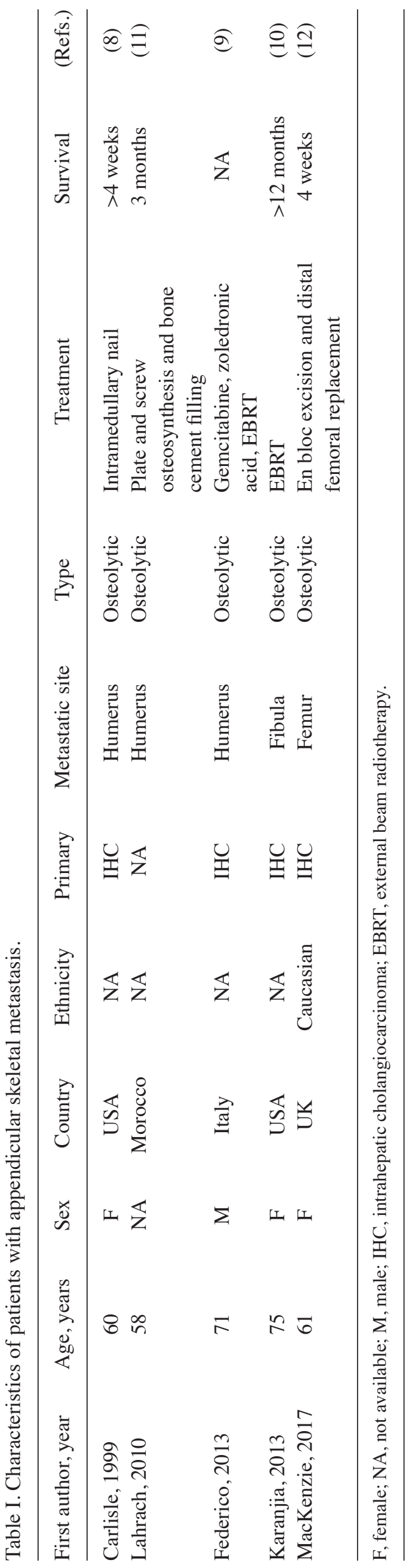


The patient received external beam radiation (30 Gy in 10 fractions) of the right scapular metastasis. One month after radiation treatment, the pain improved. Chemotherapy with carboplatin (AUC 5) and fluorouracil $\left(1,000 \mathrm{mg} / \mathrm{m}^{2}\right)$ every 4 weeks was also administered, but the tumor did not respond to the treatment and progressed after 3 cycles. The patient received palliative end-of-life care with pain control and eventually succumbed to the disease at home, 10 months after the diagnosis.

\section{Discussion}

The pattern of metastasis in CCA usually starts with lymphatic spread to the regional lymph nodes, and further spread via the hematogenous route to other organs, including the liver, lung, peritoneum and, occasionally, to the bone and brain (2-5). Bone is a common site of metastasis from several tumors, including prostate, breast and lung cancer. However, appendicular bone metastasis from CCA is a rare occurrence. The underlying reason for this rarity has not yet been elucidated.

Bone scan is not routinely performed in patients first diagnosed with CCA (6). It is only recommended for patients with bone symptoms, such as bone pain and pathological fractures. Since the majority of the patients exhibit elevated alkaline phosphatase (ALP) levels due to the primary liver pathology, ALP is not a useful marker for bone metastasis in this condition.

Spinal metastases are more frequent compared with appendicular bone metastasis. Dowsiriroj et al reported 55 cases of spinal metastases from CCA, with a median survival of only 4.0 months, despite palliative spinal surgery and radiation treatment (3).

The characteristics of the scapular mass in our patient mimicked those observed in osteosarcoma, a malignant primary bone tumor; both types of tumor are associated with severe cortical destruction of the bone and a large soft tissue mass. Furthermore, although osteosarcoma usually occurs around the knee area, the incidence of trunk and girdle tumors increases with age (7). However, the majority of adult osteosarcomas arise as secondary lesions in patients with Paget's disease of bone (7) which was not the case in this patient.

Only five cases of metastasis to the appendicular skeleton from CCA have been reported in the literature to date (Table I). The most commonly reported sites of bone metastasis are the humerus, fibula and femur (8-12). To the best of our knowledge, this is the first report of a scapular metastasis from CCA to date.

In conclusion, metastatic CCA to the bone is rare; however, oncologists should consider this as a differential diagnosis in patients who present with bone pain. Since the prognosis is extremely poor due to the minimal response to conventional chemotherapy or radiotherapy, symptom control, particularly pain, is the mainstay of treatment for improving the quality of life of the patients.

\section{Acknowledgements}

Not applicable.

\section{Funding}

No funding was received.

\section{Availability of data and materials}

All data generated or analyzed during this study are included in this published article.

\section{Authors' contributions}

PC and NT drafted the manuscript. JP interpreted the radiological data. PU provided the pathological data. JC managed the treatment for this patient and supervised the entire work. All authors have read and approved the final version of this manuscript.

\section{Ethics approval and consent to participate}

The Ethics Committee of the Faculty of Medicine of Khon Kaen University provided ethics approval under the guidelines of the Helsinki Declaration and Good Clinical Practice (HE601304).

\section{Patient consent for publication}

The legal representative of the patient in this case report provided informed consent and permission for the publication of the case details and associated images.

\section{Competing of interests}

The authors declare that they have no competing interests to disclose.

\section{References}

1. Sripa B and Pairojkul C: Cholangiocarcinoma: Lessons from Thailand. Curr Opin Gastroenterol 24: 349-356, 2008.

2. Chindaprasirt J, Sookprasert A, Sawanyawisuth K, Limpawattana P and Tiamkao S: Brain metastases from cholangiocarcinoma: A first case series in Thailand. Asian Pac J Cancer Prev 13: 1995-1997, 2012.

3. Dowsiriroj P, Paholpak P, Sirichativapee W, Wisanuyotin T, Laupattarakasem P, Sukhonthamarn K, Kosuwon W and Jeeravipoolvarn P: Cholangiocarcinoma with spinal metastasis: Single center survival analysis. J Clin Neurosci 38: 43-48, 2017.

4. Lee J, Lee SW, Han SY, Baek YH, Kim SY and Rhyou HI: Rapidly aggravated skeletal muscle metastases from an intrahepatic cholangiocarcinoma. World J Gastroenterol 21: 1989-1993, 2015.

5. Katayose Y, Nakagawa K, Yamamoto K, Yoshida H, Hayashi H, Mizuma M, Ohtsuka H, Fukase K, Onogawa T, Motoi F, et al: Lymph nodes metastasis is a risk factor for bone metastasis from extrahepatic cholangiocarcinoma. Hepatogastroenterology 59: 1758-1760, 2012.

6. National Comprehensive Cancer Network: Hepatobiliary Cancers (Version 2.2018). https://www.ncen.org/professionals/physician_ gls/pdf/hepatobiliary.pdf. Accessed July 15, 2018.

7. Ek ET, Ojaimi J, Kitagawa Y and Choong PF: Outcome of patients with osteosarcoma over 40 years of age: Is angiogenesis a marker of survival? Int Semin Surg Oncol 3: 7, 2006.

8. Carlisle RT and Roberts CS: Pathologic fracture of the humerus due to metastatic cholangiocarcinoma. South Med J 92: 1216-1219, 1999.

9. Federico A, Addeo R, Cerbone D, Iodice P, Cimmino G and Bucci L: Humerus metastasis from cholangiocarcinoma: A case report. Gastroenterology Res 6: 39-41, 2013.

10. Karanjia H, Abraham JA, O'Hara B, Shallop B, Daniel J, Taweel N and Schick FA: Distal fibula metastasis of cholangiocarcinoma. J Foot Ankle Surg 52: 659-662, 2013.

11. Lahrach K, Chbani B, Amar F, Bennani A, Marzouki A and Boutayeb F: Humerus pathological fracture revealing biliary carcinoma. Orthop Traumatol Surg Res 96: 910-912, 2010.

12. MacKenzie SA, Goffin JS, Rankin C and Carter T: Rare progression of cholangiocarcinoma: Distal femoral metastasis. BMJ Case Rep 2017: pii: bcr2016218616, 2017. 